\title{
Scaffold-mediated delivery for non-viral mRNA vaccines
}

\author{
Ruying Chen ${ }^{1} \cdot$ Hong Zhang ${ }^{1} \cdot$ Jingxuan Yan $^{1} \cdot$ James D. Bryers ${ }^{1}$
}

Received: 20 June 2018 / Revised: 22 August 2018 / Accepted: 28 August 2018 / Published online: 21 September 2018

(c) Springer Nature Limited 2018

\begin{abstract}
mRNA is increasingly being recognized as a promising alternative to pDNA in gene vaccinations. Only recently, owing to the needs of cancer immunotherapies, has the biomaterials/gene delivery community begun to develop new biomaterial strategies for immunomodulation. Here, we report a novel way to use implantable porous scaffolds as a local gene delivery depot to enhance mRNA vaccine immunization in vitro, and in vivo when compared with conventional bolus injections. We first evaluated transfection efficiencies of single-stranded mRNA condensed and charge neutralized with two lipids (Lipofectamine Messenger MAX ${ }^{\mathrm{TM}}$ LM-MM and Stemfect ${ }^{\mathrm{TM}} \mathrm{SF}$ ) and two cationic polymers (in vivo-jetPEITM, Poly ( $\beta$ amino ester)) as gene carriers. As SF demonstrated highest in vitro transfection and cell viability, it was selected for subsequent porous polymer scaffold-loading trials. Enhanced in vitro transfection of SF:mRNA nanoparticle-loaded poly (2hydroxyethyl methacrylate) (pHEMA) scaffolds was also observed with a DC2.4 cell line. Improved sustained local release and local transgene expression were also demonstrated with SF:mRNA nanoparticle-loaded pHEMA scaffolds in vivo compared with bolus injections. Our results suggest that mRNA polyplex-loaded scaffolds may be a superior alternative to either repeated bolus immunizations or ex vivo transfection cell immunotherapies.
\end{abstract}

\section{Introduction}

mRNA has emerged as a promising candidate for immunotherapy applications in recent years. First of all, mRNA has a superior safety profile over viral vectors and DNA. The genetic information carried by mRNA will be processed and expressed in the cytoplasm without the need to enter the nucleus. mRNA expression is transient thus there is no risk of genomic integration. Because there is no need to cross the nuclear membrane, mRNA is much more efficient at transfecting quiescent cells, many of which are the target cells for gene therapies and vaccines, including hepatocytes and dendritic cells (DCs). As vaccines, mRNA also serves as its own adjuvant through activation of tolllike receptors (TLR) $7 / 8$ and 3 [1-3]. This enables mRNA to trigger both arms of the humoral and cellular immunity,

Electronic supplementary material The online version of this article (https://doi.org/10.1038/s41434-018-0040-9) contains supplementary material, which is available to authorized users.

James D. Bryers

jbryers@uw.edu

1 Department of Bioengineering, University of Washington, Seattle, WA 98195-5061, USA leading to balanced and robust immune response [4]. Nucleic vaccines can elicit $\mathrm{CD} 8^{+} \mathrm{T}$-cell responses; to date, there is no clinically acceptable protein format to do this. Lastly, mRNA customization and manufacturing can be easily achieved at a low cost via in vitro transcription. This allows for personalized treatments as well as rapid development of new vaccines during pandemics.

Two major hurdles to mRNA therapies are inefficient transfection of antigen-presenting cells and in vivo instability. Numerous strategies have been developed to condense anionic nucleic acids with various cationic lipids and polymers; mostly for plasmid DNA and small siRNAs. Condensing nucleic acids with cationic molecules helps stabilize the nucleic acids while facilitating endosomal escape. Although poly (ethylenimine) (PEI) has been considered one of the most efficient polymers for gene delivery [5], lipid-based carriers have shown better capacity at facilitating mRNA transfection [6]. A class of cationic polymers, poly( $\beta$-amino esters) (PBAEs), has shown great promise as gene delivery agents owing to their facile synthesis, structural adaptability, widespread availability of monomers, biodegradability, and high transfection efficiency [7, 8]. Many systems such as lipoplexes and lipid nanoparticles (NPs) have incorporated both lipid and polymer components to optimize both transfection ability as well reduce cytotoxicity $[9,10]$. 
Also, different strategies have been developed for the administration of mRNA therapeutics [11]. Multiple clinical trials involving mRNA therapeutics are currently active, most of which rely on the return to the patient of ex vivo transfected DCs. Direct delivery of naked, uncomplexed mRNA in vivo has been administered by several routes (intradermal, intramuscular, intranasal, intraveneous, and subcutaneously) with mixed efficacy compared with complexed mRNAs $[11,12]$. To improve mRNA therapeutics, efficient and safe carrier systems are needed to overcome the numerous extra- and intracellular barriers to mRNA delivery. Only recently, owing to the needs of cancer immunotherapies, has the biomaterials/gene delivery community begun to develop new biomaterial strategies for immunomodulation. 3D porous polymer scaffolds (biodegradable or not), owing to their established success in controlled drug and cell delivery, may be ideal for manipulating directly in the body DC recruitment, antigen uptake and activation, and migration to lymph nodes. Scaffoldbased subcutaneous implants present a novel approach to mRNA delivery and may have the potential to finely tune local immune response. Porous scaffolds have been used to achieve sustained release of multiple factors, whereas providing a temporary residence and appropriate microenvironment for cell interaction [13]. Compared with bolus delivery methods, scaffold-mediated gene delivery exhibited similar to or greater expression with orders of magnitude less DNA [14]. Different forms of scaffolds and nonviral gene vectors have been developed to facilitate the immobilization, release, and uptake of gene therapeutics; mostly pDNA and siRNA [15-19]. In immunotherapy applications, the design of biomaterial scaffolds needs to be refined to better suit the different goals of immune response modulation. Although scaffolds are usually designed to promote tissue regeneration and to minimize inflammatory response in tissue engineering applications, those may not be the design criteria for vaccine applications. Research groups have released chemokines from scaffolds to recruit iDCs and other immune cells [20,21], demonstrating the potential to regulate DCs trafficking and activation in situ within scaffolds. Mooney and colleagues [22] developed a poly(lactide-co-glycolide) scaffold-based cancer vaccine that consists of granulocyte macrophage colony-stimulating factor (GM-CSF), tumor lysate (B16 melanoma), and CpGoligodeoxynucleotides as an adjuvant. Their system showed promising results in animal trials [23] and began phase I clinical trials in 2012 as the first scaffold-based cancer therapeutic. Recently, Zhang et al. [24] also demonstrated the repair of critical-sized calvarial bone defects through two-stage delivery of microRNA (miRNA) from poly(lactic-co-glycolic acid)ring mRNA to overexpress BMP-2 growth factor for bone regeneration. Not yet published (Early view), Zaitseva et al. [26] developed a collagen scaffold releasing mRNA expressing a hepatocyte growth factor for local angiogenesis. All of these above singlestranded mRNA studies were carried out as gene therapies, not as vaccine immunizations; hence results were not compared with bolus injections. So far, ours is the first study demonstrating full length, single-stranded mRNA immunization from implantable scaffolds to our knowledge.

In this study, we hypothesize that scaffold-mediated mRNA delivery in nanoparticle (polyplex) format is more efficient than naked RNA scaffold delivery or systemic injection of naked mRNA or mRNA polyplexes. We will use our porous templated scaffolds (PTS), constructs where pore size and the pore interconnects are precisely controlled throughout the entire scaffold, with both parameters being adjustable. PTS can be used as (a) stand-alone tissue regenerative constructs or (b) employed as coatings of existing indwelling devices to promote tissue integration. However, here PTS will serve as a novel highly effective vaccine depot as an alternative to systemic antigen:adjuvant injection.

We evaluated four types of polymer-based and lipidbased mRNA gene carriers (in vivo-jetPEI, Lipofectami$\mathrm{ne}^{\mathrm{TM}}$-LF-MM, PBAE, Stemfect $\left.{ }^{\mathrm{TM}}-\mathbf{S F}\right)$ as to their cytotoxicity and transfection efficiency in a range of cell lines, including DCs. We then optimized methods to maximize the mRNA:polyplex scaffold-loading efficiency and mRNA stability. Finally, mRNA-loaded (complexed or naked mRNA) $40 \mu \mathrm{m}$ pore PTS were implanted in mice and compared against subcutaneously injected mRNA (complexed or naked) for its distribution, stability, and transgene expression. We document that scaffold-mediated mRNA delivery in a nanoparticle format is more efficient than either PTS-naked RNA delivery or systemic bolus injections, demonstrating that scaffolds can be suitable tools for highly efficient mRNA delivery.

\section{Materials and methods}

\section{Plasmids and in vitro transcription of mRNA}

Plasmid DNA was prepared using the QIAprep Spin Miniprep Kit (Qiagen). Plasmid pGEM4Z-GFP-A64 (a gift from E. Gilboa, University of Miami) encoding eGFP with a synthetic poly-A tail has been previously described [27]. pGEM4Z-GFP-A64 was linearized by restriction enzyme SpeI. Linear DNA was purified using a GeneJET Gel Extraction and DNA Cleanup kit (Thermo Scientific) and used as templates for in vitro transcription using a mMESSAGE mMACHINE SP6 Kit (Ambion). No modified nucleotides were used and a standard 7methylguanosine $(\mathrm{m} 7 \mathrm{~g})$ was linked to the $5^{\prime}$-end of the mRNA through a $5^{\prime}-5^{\prime}$-triphosphate bridge (ppp). The 
resultant mRNA was purified using a RNeasy MinElute Cleanup Kit (Qiagen).

\section{Formulation and characterization of mRNA-polymer polyplexes and lipoplexes}

PEI polyplexes were formed by mixing mRNA and in vivojetPEI (Polyplus-transfection) in a $0.12: 1 \mathrm{v} / \mathrm{w}$ polymer: mRNA ratio in equal amounts of 5\% glucose solution. Linear PBAE polymer C32Ac-207 was synthesized following a protocol by Sunshine et al. [28]. Poly ( $\beta$-amino ester) (PBAE) polyplexes were prepared by mixing mRNA with the PBAE solution in $1 \mathrm{M} \mathrm{NaAc}$ solution at a N/P ratio of 10. Lipoplexes were formed by combining mRNA and liposome at a $4: 1 \mathrm{v} / \mathrm{w}$ Stemfect ${ }^{\mathrm{TM}}$ :mRNA and 1.5:1 v/w Lipofectamine $^{\mathrm{TM}}$ MessengerMax ${ }^{\mathrm{TM}}$ (LF-MM):mRNA ratio in Stemfect ${ }^{\mathrm{TM}}$ buffer and reduced serum Opti-MEM ${ }^{\mathrm{TM}}$, respectively, in accordance with the manufacturer's protocols. All polyplexes and lipoplexes were incubated for 15 min at room temperature before use. The sizes and zeta potential of poly/lipoplexes NPs were determined by dynamic light scattering (DLS) measurements using a Zetasizer Nano ZS (Malvern Pananalytical, Westborough MA). NPs were analyzed at a mRNA concentration of 10 $\mu \mathrm{g} / \mathrm{mL}$ and the mean diameters are reported as the $\mathrm{Z}$ average \pm standard deviation.

\section{Poly (2-hydroxyethyl methacrylate) (pHEMA) scaffold fabrication}

All scaffolds in this study were fabricated using a patented sphere-templating method [29] that generates 3D porous polymer scaffolds where pore size is tightly controlled, and pore interconnects are also uniform in size, with both parameters being adjustable (Supplemental Fig. 1). If the pore size is $\sim 40 \mu \mathrm{m}$, our PTS show minimal chronic inflammation while promoting healing in numerous soft and hard tissues, independent of the medical-grade polymer used. $40 \mu \mathrm{m}$ pore PTS heal owing to an early influx (13 days post implantation) of macrophages (MØ), DCs, and $\mathrm{T}$ cells, which is not observed within other pore size PTS or random pore size scaffolds that promote chronic inflammation and foreign body response. For our PTS, we have elected to use pHEMA; a hydrogel approved by the FDA for use in numerous medical devices owing to its low toxicity and for its excellent performance in histology protocols.

Poly (methyl methacrylate) (PMMA) beads $(40 \mu \mathrm{m}$ diameter) were obtained from either Microbeads AS (Norway) or Polysciences, Inc. (Warrington, PA) and sorted using an ATM Sonic model L3P Sifter (Milwaukee, WI) to obtain a desired particle size. A mold was created by placing $1 \mathrm{~mm}$ or $0.5 \mathrm{~mm}$ thick Teflon tape strips ( $2 \mathrm{~mm}$ wide) on the top of a $75 \mathrm{~mm} \times 25 \mathrm{~mm} \times 1 \mathrm{~mm}$ glass slide to serve as a retaining gasket. Sifted beads of $40 \mu \mathrm{m}$ diameter were poured into the cavity formed by the Teflon gasket. A second glass slide was fixed on top of the bead-filled cavity, thus completing the mold. Molds filled with beads were sonicated for $30 \mathrm{~min}$ in a water bath sonicator to compact the beads. Beads were then sintered to each other at their contacts points by heating at $175^{\circ} \mathrm{C}$ for $24 \mathrm{~h}$. pHEMA precursor consisted of $5 \mathrm{~mL} 2$ hydroxyethyl methacrylate (HEMA) (Polysciences), $0.23 \mathrm{~mL}$ tetraethyleneglycol dimethacrylate (Polysciences), $2.0 \mathrm{ml}$ deionized water, $3.6 \mathrm{~mL}$ ethylene glycol, and $20 \mathrm{mg}$ 2,2-dimethoxy-2-henylacetophenone (Irgacure 651) (BASF, Freeport, LA). The reaction mixture was infiltrated into the glass mold, surrounding the bead template, then degassed under vacuum for $30 \mathrm{~min}$. HEMA monomer was cross-linked into pHEMA under broad-spectrum UV for $10 \mathrm{~min}$ by free-radical polymerization.

After polymerization, the PMMA bead template surrounded by the polymerized material was removed from the mold and placed in acetone to dissolve the PMMA microspheres. The resulting porous scaffolds, composed of pHEMA gel, were cut into $3 \mathrm{~mm}$ discs using a biopsy punch. The discs were sterilized in $70 \%$ ethanol and then rehydrated in distilled water. PTS were evaluated for endotoxin using a standard limulis amebocyte lysate gel clot protocol (Lonza). All PTS batches used for in vitro and in vivo study contained endotoxin concentration $\leq 0.06 \mathrm{EU} /$ $\mathrm{ml}$. All PTS were pre-soaked in complete culture medium overnight prior to experiments.

\section{mRNA:carrier NPs loading onto scaffolds}

Preliminary data (available) indicates that a simple adsorption of mRNA (complexed or naked) from solution or indirect incorporation of mRNA within the HEMA prior to polymerization would not satisfy loading/release design parameters. As mRNA is prone to degradation at room temperature and lipoplexes are reported to be unstable in liquid suspension [30], a method of combining highconcentration mRNA lipoplex solution and lyophilization was developed to reduce the incubation time, enhance loading efficiency, and improve long-term stability of the mRNA.

To avoid nanoparticle aggregation, the optimal concentration for mRNA lipoplexes is $1-2 \mu \mathrm{g}$ mRNA/100 $\mu \mathrm{L}$ solution. Each PTS can be saturated with $\sim 100 \mu \mathrm{L}$ solution, which means one can load only $1-2 \mu \mathrm{g}$ mRNA in a single rehydration step. To maximize mRNA loading throughout a PTS, we developed a cyclical process of loading 
lyophilizing PTS (Supplemental Fig. 2A). Scaffolds were flash frozen and lyophilized after incubating with $100 \mu \mathrm{L}$ SF:mRNA nanoparticle solution for $1 \mathrm{~h}$. Scaffolds were then rehydrated in another $100 \mu \mathrm{L}$ lipoplex solution and the same incubation/lyophilization cycle was repeated for 1-4 times.

To prevent SF:mRNA NPs from aggregating during the lyophilization steps, we also carried out a re-hydrate/lyophilize cycle preliminary study with or without the cyroprotectant trehalose $(250 \mathrm{mM})$. SF:mRNA NPs alone in solution, with or without $250 \mathrm{mM}$ trehalose, were subjected to a repeated incubation/lyophilization cycle to access the effect on nanoparticle size as determined by DLS measurements.

\section{NPs loading efficiency and NP scaffold distribution}

The amount of RNA loaded into the PTS was determined by closing the mass balance for the total amount of RNA released from the scaffolds. After loading, scaffolds were submerged in $100 \mathrm{mg} / \mathrm{mL}$ heparin and incubated for $10 \mathrm{~min}$ at room temperature to displace the mRNA from the SF NPs. The resultant solution was then stained with Quant$\mathrm{iT}^{\mathrm{TM}}$ RiboGreen ${ }^{\circledast}$ assay (Invitrogen) in accordance to manufacture's protocol. Fluorescence intensity was measured with plate reader and converted with standard curve into mass mRNA.

To visualize the distribution of SF-mRNA NPs, a crosssection of the scaffold after NPs loading was imaged on a Zeiss Axio Observer Z1 fluorescence microscope with an ApoTome optical sectioning attachment.

\section{Cell culture}

All cell culture medium and reagents were obtained from Gibco unless otherwise specified. The BHK-21 hamster fibroblast cell line (ATCC) was maintained in Eagle's Medium supplemented with $10 \%$ fetal bovine serum (FBS) and $1 \%$ penicillin-streptomycin.

The DC2.4 murine dendritic cell line (a gift from K.L. Rock, University of Massachusetts Medical School) was maintained in RPMI 1640 containing L-glutamine supplemented with $10 \mathrm{mM}$ 4-(2-hydroxyethyl)-1-piperazineethanesulfonic acid, $0.1 \mathrm{mM}$ non-essential amino acids, $55 \mu \mathrm{M}$ 2-mercaptoethanol, $10 \%$ FBS, and $1 \%$ penicillin-streptomycin.

The JAWsII murine dendritic cell line (ATCC) was maintained in alpha minimum essential medium supplemented with $1.5 \mathrm{~g} / \mathrm{L}$ sodium bicarbonate, $2 \mathrm{mM}$ L-glutamine, $5 \mathrm{ng} / \mathrm{ml}$ GM-CSF, $20 \%$ FBS, and $1 \%$ penicillinstreptomycin.

Murine bone marrow-derived dendritic cells (BMDCs) were obtained and isolated from the femurs and tibiae of 6-
8 week-old female or male C57BL/6 mice. Cells were cultured in Petri dishes in $10 \mathrm{ml}$ complete medium containing $20 \mathrm{ng} / \mathrm{ml}$ granulocyte macrophage colonystimulating factor (GM-CSF, Peprotech, Rocky Hill, NJ). Ten milliliter fresh medium containing $20 \mathrm{ng} / \mathrm{ml} \mathrm{GM-CSF}$ was added on Day 3, and medium was changed on Day 5. Cells were harvested and used for experiments on Day 7. All procedures involving animals were approved by the University of Washington Institutional Animal Care and Use Committee.

\section{mRNA in vitro transfection}

The ability of cationic polymers and liposomes to mediate mRNA transfection in vitro was evaluated in baby hamster kidney fibroblast cell lines, BHK-21; murine dendritic cell lines, DC2.4; JAWsII; and murine primary BMDCs. For all transfection experiments, cells were plated in 24-well plate $24 \mathrm{~h}$ prior to exposure to mRNA/carriers. Polyplexes and lipoplexes were formulated as described above and added to the cells at $1 \mu \mathrm{g} \mathrm{mRNA} /$ well. Cell medium was added to each well to obtain a total volume of $1 \mathrm{~mL} /$ well and the poly/lipoplexes were allowed to incubate with cells for 24 or $48 \mathrm{~h}$. After this time, the cells were washed and collected in phosphate-buffered saline (PBS)-based cell dissociation buffer (Invitrogen) or $0.25 \%$ trypsinethylenediaminetetraacetic acid (EDTA) and resuspended in Dulbecco's phosphate-buffered saline (DPBS) containing $2 \% \mathrm{FBS}$ and $0.2 \mu \mathrm{g} / \mathrm{mL}$ propidium iodide (Invitrogen). To determine cell viability and GFP expression levels, 10,000 events per sample gated on single cells were acquired on a BD LSRII flow cytometer (BD Biosciences) and analyzed using FlowJo software. In some experiments, cells were washed once with DPBS and then fixed with $4 \%$ paraformaldehyde for $15 \mathrm{~min}$, then imaged on a Zeiss Axio Observer Z1 fluorescence microscope with an ApoTome optical sectioning attachment.

DC2.4 cells were used for scaffold-mediated mRNA transfection in vitro. Cells were maintained in complete medium in T75 flasks until the experiment. Cells were detached with $0.25 \%$ Trypsin-EDTA, centrifuged and resuspended in fresh medium to a concentration of $10^{7}$ cells/ $\mathrm{mL}$. Lyophilized mRNA-loaded scaffolds were rehydrated in complete medium for $1 \mathrm{~h}$ before cell seeding at $37^{\circ} \mathrm{C}$. To seed cells into scaffold, sheets of sterile Kimwipes ${ }^{\circledR}$ were placed underneath the scaffold to draw the cell solution through the scaffold by capillary force. Hundred microliters of cell solution was added onto the scaffold in several applications until fully absorbed. To observe the spatial distribution of transfected cells in a PTS after $24 \mathrm{~h}$ culture, PTS along with seeded cells were fixed and imaged with a Zeiss Axio Observer Z1 fluorescence microscope with an ApoTome optical sectioning attachment using a $\times 20$ 

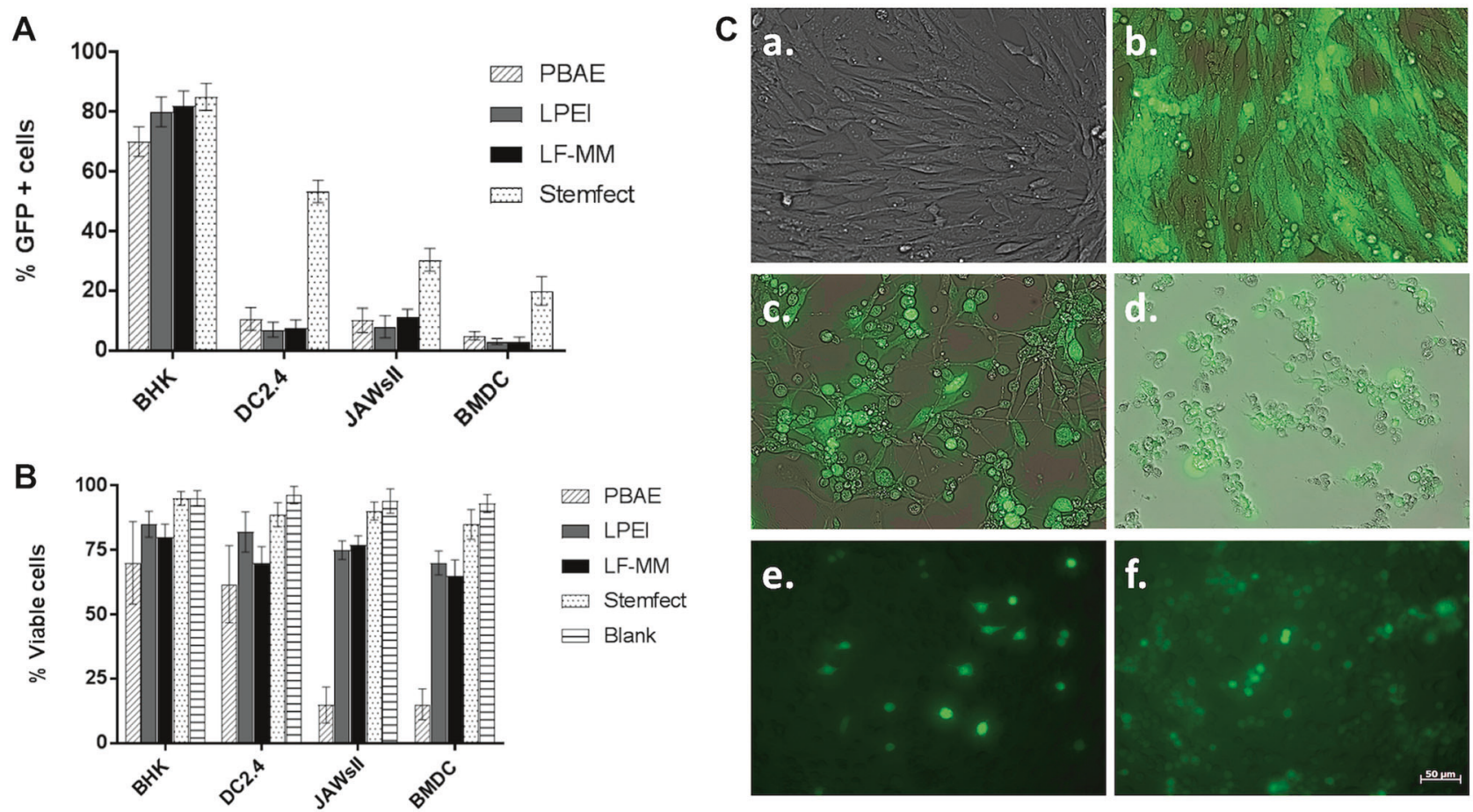

Fig. 1 Different mRNA carriers impact on A. in vitro transfection efficiency as \% GFP-positive cells and B. cell viability after $24 \mathrm{~h}$ exposure to poly/lipoplexes. Data are from experiments conducted in triplicate \pm standard deviation. Error bars are s.d. C. Cell morphology and GFP expression upon in vitro transfection with mRNA poly/

objective. Some scaffolds were also embedded in OCT compound and frozen for cryosection. Flow cytometry analysis of transfected cells within a PTS was not carried out because: (1) removing cells from the PTS would destroy any information about spatial distribution of transfected cells, (2) removing cells from PTS is very inefficient, and (3) homogenizing the pHEMA PTS to extract cells would contaminate samples with PTS debris that is autofluorescent.

\section{Scaffold-mediated mRNA delivery in vivo}

All animal experiments adhered to federal guidelines and were approved by the University of Washington Institutional Animal Care and Use Committee. Groups of C578L/ $6 \mathrm{~J}$ male and female (1:1) mice (Jackson Laboratory, 68 weeks old. $n=3$ mice/group) were used for this study. Statistical power analysis of pilot and published studies was used to determine the number of replicates $(N=3)$ sufficient to show a statistically significant effect with $80 \%$ power in groups for the GFP expression to be measured here. Animals were assigned randomly to receive the various mRNA treatments by animal care personnel, thus removing the investigator from the selection process.

After anesthetizing with $2 \%$ isoflurane, PTS loaded with either naked mRNA or SF:mRNA NPs were implanted

lipoplexes. BHK fibroblast cells upon $24 \mathrm{~h}$ exposure to a blank control with no mRNA, PBS only; b SF:mRNA nanoparticles; $\mathbf{c}$ in vivo-jetPEI:mRNA, d PBAE:mRNA. JAWsII cells exposed to e LF-MM: mRNA; and $\mathbf{f} S F$ :mRNA nanoparticles. mRNA in each assay was $1 \mu \mathrm{g}$. Scale bar is $50 \mu \mathrm{m}$

subcutaneously in the dorsal right flank. For mice without scaffolds, $200 \mu \mathrm{L}$ naked mRNA or SF:mRNA NPs were injected subcutaneously at the same location. Mice injected with $200 \mu \mathrm{L}$ PBS alone were used as negative controls. In all cases, total amount of mRNA delivered by implantation or injection was $4 \mu \mathrm{g}$.

To image mRNA distribution in mice, mRNA was labeled with Cy5 using a LabelIT Tracker Kit (MirusBio) at a $0.5 \mathrm{v} / \mathrm{w}$ ratio of LabelIT Tracker Reagent to mRNA in accordance with the manufacturer's protocol. Mice were anesthetized with isoflurane and imaged for Cy5 fluorescence using a Xenogen IVIS200 Spectrum Imager. Image analysis was performed using Living Image software (Caliper).

At 24 or $72 \mathrm{~h}$ post implantation/injection, animals were euthanized by $\mathrm{CO}_{2}$. Implants along with incorporated tissue were excised and imaged with Xenogen IVIS200 Spectrum Imager for GFP expression. Following this, the explanted PTS and their ingrown tissue were digested into single cell suspensions using a collagenase solution (Worthington, 250 $\mathrm{U} / \mathrm{ml}$ ) that was agitated at $37{ }^{\circ} \mathrm{C}$ for $45 \mathrm{~min}$. The cell suspensions were then poured through a $40-\mu \mathrm{m}$ cell strainer to isolate cells from scaffold debris. Cells were then labeled with the viability stain propidium iodide and antibody CD11c. In total, 10,000 live single cell events per sample were acquired on a BD FACScan flow cytometer (BD 

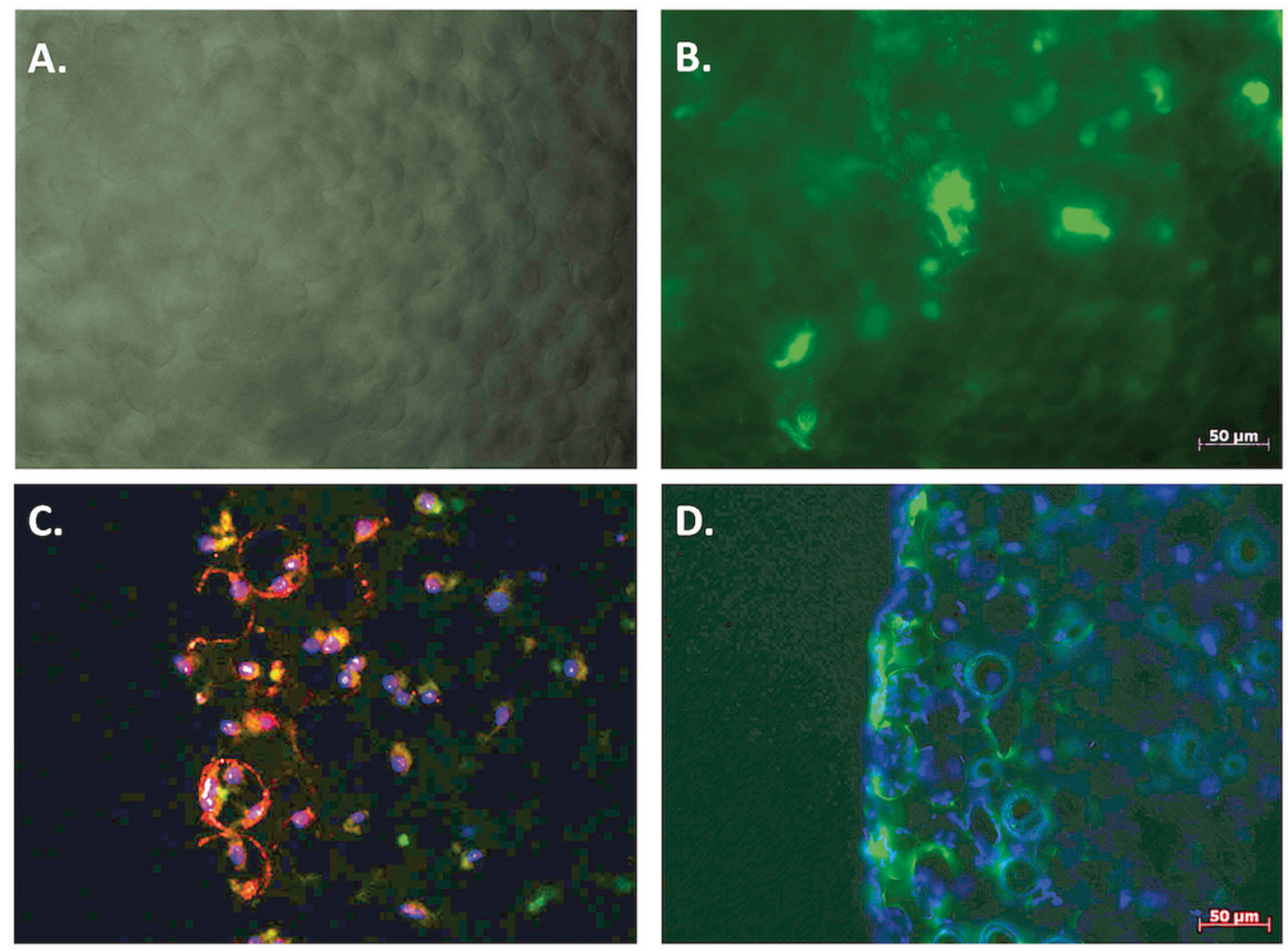

Fig. 2 DC2.4 in vitro transfection within $40 \mu \mathrm{m}$ pore pHEMA scaffolds containing SF:mRNA nanoparticles ( $4 \mu \mathrm{g}$ mRNA) after $24 \mathrm{~h}$. A. no mRNA control; empty PTS only, B. DC2.4 $24 \mathrm{~h}$ post transfection. Cross-section images of $40 \mu \mathrm{m}$ pHEMA PTS $24 \mathrm{~h}$ after DC2.4

transfection C. Cy5-labeled SF:mRNA nanoparticle uptake, D. GFP expression. Cell nucleus was stained with DAPI in C and D. Scale bar is $50 \mu \mathrm{m}$

Biosciences) and analyzed using FlowJo software (TreeStar).

SF:mRNA NPs were more efficient in transfecting DC2.4 cell lines $(53.3 \% \mathrm{GFP}+)$ than JAWsII cell lines $(30.4 \%$ GFP +) (Fig. 1A).

Cytotoxicity of the polymer or lipid carriers was also

\section{Statistics}

Statistical analysis was performed using correlation functions for two-tailed Student's $t$ test or two-way analysis of variance with the software GraphPad Prism.

\section{Results}

\section{mRNA in vitro transfection with various compounds}

The ability of cationic polymers and liposomes to mediate mRNA transfection in vitro was evaluated in a various cell lines and primary cells. Although all reagents exhibited comparable abilities in transfecting BHK fibroblast cells, most of them were not efficient at facilitating mRNA transfection in DCs. Twenty-four hours after vector exposure, transfection rates were lower than 10 and $3 \%$ for DC lines and primary DC, respectively. In contrast, transfection efficiency with SF:mRNA NPs in the same DC cells were from 3 to 6 times higher than the other carriers tested. Also, evaluated with flow cytometry. Although cells transfected with commercial standard LF-MM had $70 \sim 80 \%$ viability after $24 \mathrm{~h}$, over $90 \%$ cells transfected with Stemfect ${ }^{\mathrm{TM}}$ were still viable $24 \mathrm{~h}$ later, which was approximated the same as the control group that received PBS only (Fig. 1B). In more robust BHK cell lines, such cytotoxicity effects are reflected in morphological changes. Cells transfected with Stemfect ${ }^{\mathrm{TM}}$ lipoplex showed the typical elongated morphology for BHK cells. Parallel cultures of BHK, $24 \mathrm{~h}$ after exposure to in vivo-jetPEI and PBAE vectors produced cells with more rounded shapes along with cell clumps. Such morphological changes were accompanied by slower growth or cell death as evidenced in lower cell density (Fig. 1C).

\section{Effects of re-hydrate/lyophilize cycles on SF:mRNA NPs and subsequent transfection efficiency}

Lyoprotectant trehalose was incorporated in the SF:mRNA nanoparticle preparation to prevent particle aggregation and to retain mRNA functionality during lyophilization cycles. 
Fig. 3 In vivo tissue distribution and quantitation of Cy5-labeled SF:mRNA nanoparticles originally in $40 \mu \mathrm{m}$ PTS. Delivered mRNA is all cases was $4 \mu \mathrm{g}$. Top: IVIS live animal imaging on Day 1 and Day 3.

Mice were either implanted with $40 \mu \mathrm{m}$ pore pHEMA PTS loaded with either naked mRNA or SF: mRNA nanoparticles, or received the same amount of mRNA (either naked mRNA or SF:mRNA nanoparticles) via subcutaneous injection; Bottom: Average Cy5 fluorescence signal measured by IVIS; $(N=3)$ errors bars are s.d

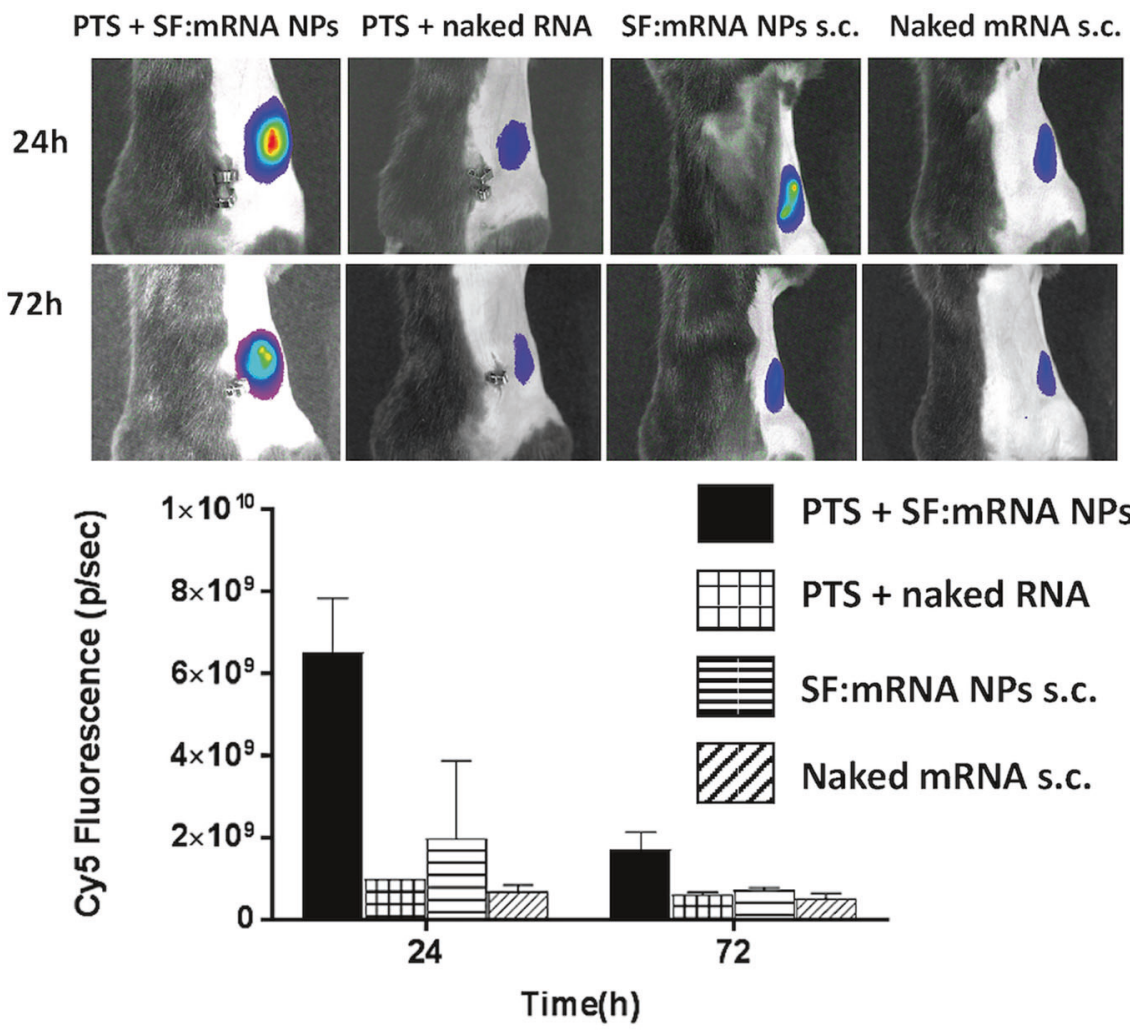

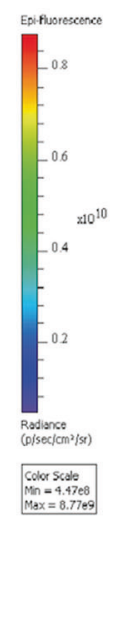

Particle size was measured $10 \mathrm{~min}$ post nanoparticle formation and after each lyophization/rehydration cycle. The addition of $250 \mathrm{mM}$ trehalose solution did not affect the particle size immediately after lipoplex formation. Moreover, it significantly prevented particle aggregation during lyophilization cycles (Supplementary Figure 2B). Lipoplexes formulated with trehalose retained an average size of $<300 \mathrm{~nm}$ after the first two lyophilization cycles, and $<500$ $\mathrm{nm}$ after the third cycle. In contrast, formulation without any cryoprotectant quickly aggregated SF:mRNA NPs into aggregates $\sim 2 \mu \mathrm{m}$ in diameter after the first lyophilization/ rehydration cycle.

It is generally accepted that lipoplexes size affects the endocytosis pathway and resulting intracellular trafficking in cells [31]. To investigate how the functionality of the SF: mRNA nanoparticle correlates to the size change, their bioactivity was evaluated by in vitro transfection of BHK cells (Supplementary Figure 3). Results show that an increase in particle size lead to a decrease in lipoplexes mRNA transfection efficiency. Without lyoprotectant, the mRNA lipoplexes lost over $90 \%$ transfection efficiency after the first freeze-dry cycle. In contrast, with the addition of trehalose, the particles retained 80 and $65 \%$ of transfection capacity after two and three freeze-drying cycles compared with freshly prepared particles, respectively.

\section{Loading efficiency and mRNA NPs distribution}

A repeated series of lyophilization-rehydration steps was developed to load SF:mRNA NPs into $40 \mu \mathrm{m}$ pore size PTS. Repeatedly, $83.7 \pm 9.2 \%$ SF:mRNA NPs adhered to the scaffold $(\sim 4 \mu \mathrm{g}$ mRNA); the lyophilization-rehydration cyclical process also lead to a more uniform nanoparticle distribution throughout the scaffold compared with single PTS rehydration step (Supplementary Figure 4).

\section{In vitro scaffold-mediated mRNA transfection}

An in vitro scaffold-mediated mRNA transfection was carried out using DC2.4 murine DCs. SF:mRNA NPs were loaded onto $40 \mu \mathrm{m}$ PTS by way of the lyophilizationrehydration method. Whereas SF:mRNA NPs formulated without lyoprotectant produced no mRNA transfection, trehalose formulated SF:mRNA NPs successfully transfected DC2.4 cells seeded within the $40 \mu \mathrm{m}$ PTS (Fig. 2). Cross-section images of the scaffold documents Cy5labeled mRNA uptake (Fig. 2C) and the spatial distribution of GFP-expressing cells (Fig. 2D). Although the seeding procedure allowed cell infiltration throughout the scaffold, most of the in vitro mRNA uptake and GFP protein expression was observed on the outer scaffold layers, which may be due to nutrient gradients (e.g., oxygen) 
Fig. 4 IVIS imaging and quantitation of local GFP expression from scaffolds and contained tissue at 24 A. and 72 B. hours; each lane represents samples from a different mouse. Delivered mRNA is all cases was $4 \mu \mathrm{g}$. Experimental groups include $40 \mu \mathrm{m}$ pHEMA PTS control (PTS only), pHEMA PTS with SF:mRNA nanoparticles (PTS SF:mRNA nanoparticles), pHEMA PTS with naked mRNA (PTS-naked mRNA), SF:mRNA nanoparticles subcutaneous bolus injection (SF:mRNA s.c.), and naked mRNA subcutaneous bolus injection (naked mRNA $\mathrm{s}$. c.). $(N=3)$. C. Average GFP fluorescence signal measures by IVIS; $(N=3)$ error bars are s.d

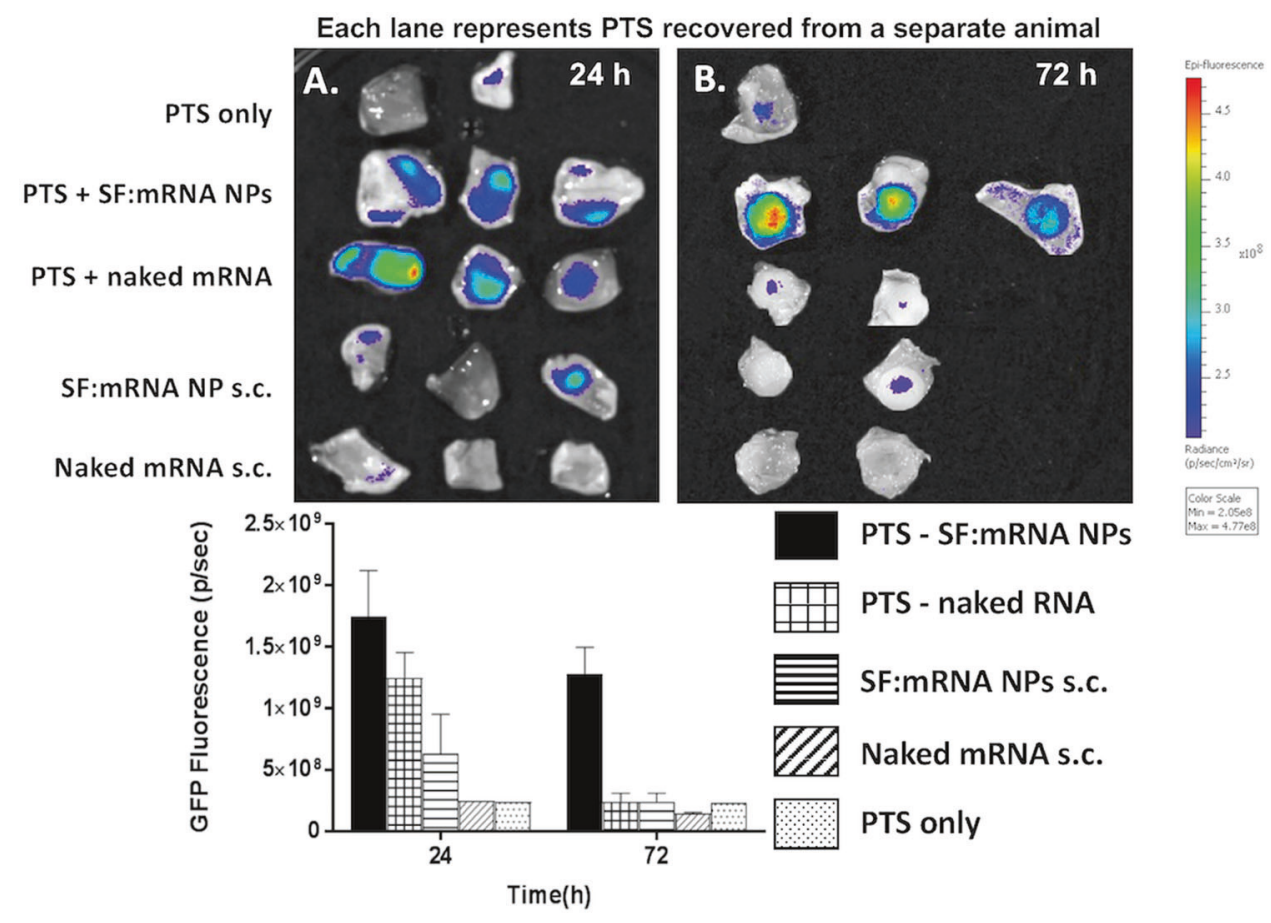

causing growth rate gradients within the scaffolds. Overall, these results indicate that SF:mRNA NPs deposited on pHEMA scaffolds retained the capacity to mediate mRNA intracellular transfection.

\section{In vivo imaging of mRNA vector retention}

Live animal imaging was used to monitor the in vivo tissue distribution of SF:mRNA NPs containing fluorescent Cy5tagged mRNA. pHEMA $40 \mu \mathrm{m}$ scaffolds loaded with Cy5labeled mRNA encoding for GFP (as SF:mRNA NPs or free mRNA) were implanted in mice as described above. Naked mRNA in solution was administered to mice via subcutaneous injection for comparison. Consistent with our previous hypothesis, mRNA delivered from implanted scaffolds exhibited prolonged release compared with either naked RNA release from PTS or bolus injection delivery (Fig. 3). Twenty-four hours and $72 \mathrm{~h}$ post implantation/ injection, the amount of Cy5-labeled mRNA retained on scaffolds is more than three times that retained at an injection site. Also, at each time point, PTS containing SF: mRNA NPs have 2.5-6.7 times stronger signals than PTS containing naked mRNA, suggesting the lipoplexes successfully protected mRNA from degradation and improved stability in vivo.

\section{In vivo scaffold-mediated transfection}

To investigate if higher mRNA local retention leads to higher transfection efficiency, local GFP expression was compared for explanted scaffolds and tissue contents (Fig. 4). Twenty-four hours post implantation/injection, GFP expression was detected on both scaffold groups (naked or SF:mRNA NPs), whereas almost no signal was detected from either bolus suspension injection group. At Day 3, GFP expression was equivalent to the $24 \mathrm{~h}$ level, indicating a more sustained GFP expression was achieved with PTS-mediated delivery of SF:mRNA NPs. Flow cytometry analysis of cells extracted from the implants showed both PTS-based local delivery of SF:mRNA NPs (Cy5 stained mRNA; Fig. 5) lead to an enhanced and more persistent expression of mRNA-GFP locally (Supplemental Fig. 5). On Day 3, 15\% cells analyzed from $40 \mu \mathrm{m}$ pHEMA PTS presenting SF:mRNA NPs showed Cy5 $(+)$ mRNA uptake (Fig. 5), in comparison with 6\% from bolus delivery group. GFP expression (Supplemental Fig. 5) similarly showed at Day $119 \%$ of cells dropping to $9 \%$ of cells on Day 3 expressing GFP, while GFP expression for all other delivery platforms was between 2.0 and $6.0 \%$.

Our previously published work [32] showed that at Day $315 \%$ of cells in an explanted $40 \mu \mathrm{m}$ PTS were CD11b + CD11c- macrophages and 8\% CD11c + dendritic cells, of which $\sim 10 \%$ were major histocompatibility complex (MHC)-II + CD86 + mature DCs. Although the macrophage population did not vary as time progressed, DC populations dropped, suggesting a migration to the lymph nodes. However, in this study flow cytometry (Supplementary Figures 6 and 7) and IVIS fluorescent imaging of explanted lymph nodes (Supplemental Figure 8) indicated lymph node homing did not occur. 
Fig. 5 In vivo local uptake at site of PTS implantation of Cy5labeled mRNA on Day 1 and Day 3 analyzed by flow cytometry. Delivered mRNA is all cases was $4 \mu \mathrm{g}$. Displayed experimental groups include 40 $\mu \mathrm{m}$ pore PTS with SF:mRNA nanoparticles (PTS SF:mRNA nanoparticles), PTS with naked mRNA (PTS-naked mRNA), SF:mRNA nanoparticles subcutaneous bolus injection (SF:mRNA s.c.), and naked mRNA subcutaneous bolus injection (naked mRNA s.c.)
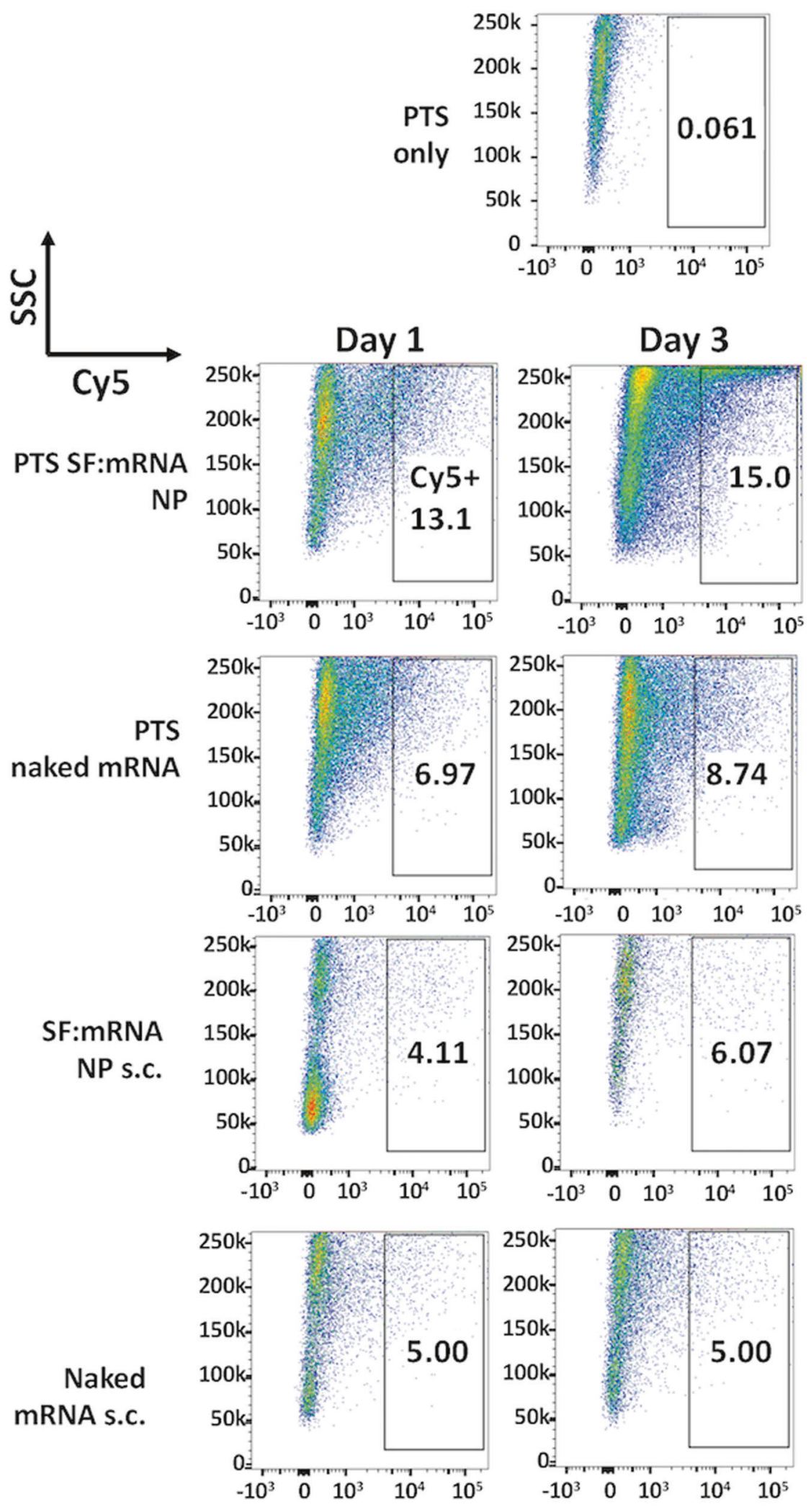


\section{Discussion}

mRNA is prone to degradation by various of enzymes in vivo. Multiple studies have reported that naked mRNAs exhibit poor ability to transfect cells and have very short half-life in vivo [11]. This enzymatic liability poses a great barrier to the development of mRNA therapeutics. Efficient transfection of DCs provides a powerful tool for vaccine development, cancer immunotherapy, and autoimmune disease therapies [33]. Although DCs remain one of the most difficult cells to transfect, promising new methods combining cationic polymers or lipids with mRNA have recently been successful. To expand the scope of applications with DCs, especially for vaccine development, novel methods of transfecting into DCs have been, and will need to be, improved. The first objective of this study was to develop mRNA-poly/lipoplex nanoparticle formulation suitable for DCs transfection. The second objective of this study was to create gene-activated scaffolds optimized for local mRNA acquisition. This is done by incorporating the mRNA NPs onto pHEMA-based scaffolds with properties optimized for DCs activation [32].

In this study, we selected four types of commonly used polymer- and lipid-based gene carriers, in vivo-jetPEI, PBAE, LF-MM ${ }^{\mathrm{TM}}$, and Stemfect ${ }^{\mathrm{TM}}$, to compare their transfection efficiency as well as cytotoxicity to different cell types. In general, it was observed that lipid-based LF$\mathrm{MM}$ and Stemfect ${ }^{\mathrm{TM}}$ carriers are more effective than polymer based in vivo-jetPEI and PBAE at mediating mRNA expression in vitro, which is consistent with most current studies in mRNA delivery. It has been reported by some groups $[34,35]$ that release of mRNA from a carrier in the cytoplasm is necessary for ribosomal recognition. Therefore, the binding strength between mRNA and the cationic polymer or lipid will greatly affect mRNA expression efficiency. Bettinger et al. [34] demonstrated that singlestranded mRNA binding to cationic polymers is stronger than pDNA binding. Polymers with larger molecular weight have higher affinity to mRNA than polymers with smaller molecular weight. This explains why polymers such as PEI $(22 \mathrm{kDa}, 25 \mathrm{kDa})$ and poly(L-lysine), which are preferred for DNA delivery owing to their great stability, led to poor results for mRNA delivery. The Stemfect ${ }^{\mathrm{TM}}$ :mRNA transfection efficiency in DCs was assessed in a similar study conducted by Phua et al. [11]; over 60\% BMDC were expressing GFP protein 8 h post culture with the complex. In our study, although SF:mRNA NPs had a varied ability to mediate mRNA expression in different dendritic cell lines (DC2.4 > JAWsII > BMDC), its overall reported transfection rate is notably higher than other carriers. PBAE polyplexes showed comparable transfection to the commercially available LF-MM and Stemfect ${ }^{\mathrm{TM}}$. However, PBAE also exhibited higher cytotoxicity to cells, which is commonly seen with cationic polymers. PEG and various lipids have been used to shield the charge on the surface of cationic polymers and thus to reduce cytotoxicity [36].

One hurdle to mRNA scaffold-based delivery is maintaining mRNA stability and bioactivity during the loading process. In many applications, protein or nucleic acids are coated onto scaffolds through physical adsorption. However, most mRNA will lose its bioactivity after prolonged incubation under room temperature or $37^{\circ} \mathrm{C}$.

As our in vitro study revealed, lipid-based carriers were more efficient at mRNA delivery. We experimented with different strategies to improve physical adsorption or embedding of the lipoplexes, including copolymerize HEMA with negatively charged methacrylic acid or applying thin coatings of fibrin/lipoplex mixture over the surface of scaffold. Although a negatively charged surface greatly improved loading efficiency, the amount of charge required induced significant cytotoxicity and scaffold swelling in vivo. Fibrin coating rendered a soft layer on the scaffold surface that was not uniform. Although some experiments with fibrin-coated scaffolds produced positive results, the overall transfection consistency was poor (data not shown). Consequently, a repeated sequence of physical adsorption of SF:mRNA NPs followed with a lyophilization step was used for mRNA loading. Although this is a common method used to load therapeutics onto scaffolds, additional optimization was needed for SF:mRNA NPs, which are prone to degradation and aggregation. The use of a lyoprotectant such as glucose or trehalose was previously reported [30, 37]. In our study, the addition of trehalose successfully maintained mRNA bioactivity and minimized nanoparticle aggregation during the adsorption and lyophilization cycles.

Scaffold-mediated delivery differs from solutionmediated in that cells are seeded on top of the genetic particles that have been previously immobilized onto the scaffold rather than adding particles to previously seeded cells. It provides a simple method to locally deliver genetic materials from the surface of a porous implant. To date, a multitude of gene-activated scaffolds for tissue engineering have been described such as those incorporating PEI in both modified [38] and unmodified forms [39], chitosan [40], poly-L-lysine [41], cationic dextrans [42], and lipid-based systems [13] to name a few. All of these systems are designed for sustained release of plasmid DNA or siRNA, whereas our study is the first to report an mRNA activated scaffold. With a mouse subcutaneous implant model, we demonstrated that both (a) optimized mRNA formulation and (b) vector release platform (injection vs. scaffold) played important roles in promoting sustained mRNA local release and expression. Lipoplex-loaded scaffolds outperformed other control groups (naked RNA loaded scaffolds and subcutaneous injections) in all aspects, including 
prolonged local release of mRNA, improved local mRNA uptake by cells, and increased GFP transgene expression at implantation site. A few reasons contributing to the enhanced performance from scaffold-mediated delivery in vivo may be: (a) nanoparticle formulation protected mRNA from enzymatic degradation while facilitating transfection; (b) immobilization process preserves the NP size observed in solution and reduces exposure of the particles to various enzymes in vivo, which often induces aggregation and de-complexation [43]; (c) biomaterial scaffolds loaded with NPs were able to maintain an elevated concentration of gene payload locally, which increases the probability of cellular internalization; and (d) surgical implantation and subsequent immune response intensify levels of antigen-presenting cells at the wound site. Interestingly, although most current literature reported that naked mRNA is superior to NPs in transfection via subcutaneous bolus delivery [11], we have observed significantly enhanced expression from nanoparticle transfection at the scaffold implantation site. It is hypothesized that cells present at subcutaneous space are mostly MHC-II-negative cells, therefore are less efficient at taking up NPs through non-specific endocytic mechanisms [11]. As we have observed accumulation of MHC-II-positive macrophages at the scaffold post implantation [32], it may explain the enhanced efficiency for nanoparticle transfection from subcutaneous porous implants.

Vaccine delivery is one of the potential applications for this subcutaneous porous scaffold platform. Efficient DC transfection is often essential for developing potent vaccines and it is usually achieved by NPs trafficking to draining lymph nodes. The number of DCs naturally occurred at implantation site is low and the duration is transient [32]. So, it was not surprising to find out that although DC transfection was demonstrated with high efficiency in vitro, minor DC-specific transfection was observed at the implantation site in vivo with no lymph node homing detected. This can be improved in future studies by enriching and programming DCs at the implantation site with co-delivered cytokines and adjuvants. In addition, when delivered from a scaffold, particle-and-cell-substrate interactions influence the uptake and expression of mRNA vectors. It would be interesting to explore how the molecular chemistry of the scaffold materials will influence the endocytic behavior and transfection ability in future work.

\section{Conclusion}

In this study, we evaluated various gene carriers for mRNA and optimized a precision porous scaffold system for mRNA localized delivery. Results indicated that with optimized formulation and loading, mRNA stability and bioactivity was preserved. Scaffold-mediated transfection was successfully demonstrated in vitro and in vivo. In vivo results suggested that both lipoplex formulation and local immobilization of mRNA via scaffolds were important to mRNA-sustained delivery and transfection. Lipoplexesloaded scaffolds excelled other control groups (naked RNA loaded scaffolds and subcutaneous injections) in all aspects, including prolonged local release of mRNA, improved local mRNA uptake by cells, and increased GFP transgene expression at implantation site. These results demonstrate that scaffold-based delivery has advantages over suspension bolus injection delivery and can be potentially used as an mRNA therapeutics delivery platform.

Acknowledgements This work was supported by the National Institutes of Health/NIAID (5R01AI074661 and 2R56AI074661-06).

Author contributions Ruying Chen designed and executed the study, analyzed and interpreted the data, and drafted and revised the manuscript. Hong Zhang executed the PBAE study, interpreted the data, and revised the manuscript. Jingxuan Yan executed portions of the study, interpreted the data, and revised the manuscript.

\section{Compliance with ethical standards}

Conflict of interest The authors declare that they have no conflict of interest.

\section{References}

1. Heil F, Hemmi H, Hochrein H, Ampenberger F, Kirschning C, Akira $\mathrm{S}$, et al. Species-specific recognition of single-stranded rna via toll-like receptor 7 and 8. Science. 2004;303:1526-9.

2. Diebold SS, Kaisho T, Hemmi H, Akira S, Reis E, Sousa C. Innate antiviral responses by means of TLR7-mediated recognition of single-stranded RNA. Science. 2004;303:1529-31.

3. Alexopoulou L, Holt AC, Medzhitov R, Flavell RA. Recognition of double-stranded RNA and activation of NF- $\kappa \mathrm{B}$ by Toll-like receptor 3. Nature. 2001;413:732-8.

4. Fotin-Mleczek M, Duchardt KM, Lorenz C, Pfeiffer R, OjkićZrna S, Probst J, et al. Messenger RNA-based vaccines with dual activity induce balanced TLR-7 dependent adaptive immune responses and provide antitumor activity. J Immunother. 2011;34:1-15.

5. Godbey WT, Wu KK, Mikos AG. Poly(ethylenimine) and its role in gene delivery. J Control Release. 1999;60:149-60.

6. Yamamoto A, Kormann M, Rosenecker J, Rudolph C. Current prospects for mRNA gene delivery [Internet]. Eur J Pharm Biopharm. 2009;71:484-9.

7. Gao Y, Huang J-Y, O'Keeffe Ahern J, Cutlar L, Zhou D, Lin F-H, et al. Highly branched Poly( $\beta$-amino esters) for non-viral gene delivery: high transfection efficiency and low toxicity achieved by increasing molecular weight. Biomacromolecules. 2016;17:36407.

8. Koo H, Jin Gwoo, Kang H, Lee Y, Nam HY, Jang Hsuk, et al. A new biodegradable crosslinked polyethylene oxide sulfide (PEOS) hydrogel for controlled drug release. Int J Pharm. 2009;374:5865 . Jun 5 
9. Persano S, Guevara ML, Li Z, Mai J, Ferrari M, Pompa PP, et al. Lipopolyplex potentiates anti-tumor immunity of mRNA-based vaccination. Biomaterials. 2017;125:81-9.

10. Su X, Fricke J, Kavanagh DG, Irvine DJ. In vitro and in vivo mRNA delivery using lipid-enveloped $\mathrm{pH}$-responsive polymer nanoparticles. Mol Pharm. 2011;8:774-87.

11. Phua KKL, Leong KW, Nair SK. Transfection efficiency and transgene expression kinetics of mRNA delivered in naked and nanoparticle format. J Control Release. 2013;166:227-33.

12. Hajj KA, Whitehead KA. Tools for translation: non-viral materials for therapeutic mRNA delivery. Nat Rev Mater. 2017;2:17056.

13. Xie Y, Yang ST, Kniss DA. Three-dimensional cell-scaffold constructs promote efficient gene transfection: implications for cell-based gene therapy. Tissue Eng. 2001;7:585-98.

14. Jang J-H, Bengali Z, Houchin TL, Shea LD. Surface adsorption of DNA to tissue engineering scaffolds for efficient gene delivery. $\mathrm{J}$ Biomed Mater Res Part A. 2006;77A:50-8.

15. O'Rorke S, Keeney M, Pandit A. Non-viral polyplexes: scaffold mediated delivery for gene therapy. Prog Polym Sci. 2010;35:441-58.

16. Kim YM, Park MR, Song SC. Injectable polyplex hydrogel for localized and long-term delivery of siRNA. ACS Nano. 2012;6:5757.

17. Wang LL, Burdick JA. Engineered hydrogels for local and sustained delivery of RNA-interference therapies. Adv Healthc Mater. 2017;6:62017.

18. Nguyen K, Dang PN, Alsberg E. Functionalized, biodegradable hydrogels for control over sustained and localized siRNA delivery to incorporated and surrounding cells. Acta Biomater. 2013;9:4487.

19. Ma Z, Yang C, Song W, Wang Q, Kjems J, Gao S. Chitosan hydrogel as siRNA vector for prolonged gene silencing. J Nanobiotechnol. 2014;12:23.

20. Stachowiak AN, Irvine DJ. Inverse opal hydrogel-collagen composite scaffolds as a supportive microenvironment for immune cell migration. J Biomed Mater Res Part A. 2008;85:815-28.

21. Shea LD, Smiley E, Bonadio J, Mooney DJ. DNA delivery from polymer matrices for tissue engineering. Nat Biotechnol. 1999;17:551-4.

22. Ali OA, Mooney DJ. Sustained GM-CSF and PEI condensed pDNA presentation increases the level and duration of gene expression in dendritic cells. J Control Release. 2008;132:273-8.

23. Ali OA, Huebsch N, Cao L, Dranoff G, Mooney DJ. Infectionmimicking materials to program dendritic cells in situ. Nat Mater. 2009;8:151-8.

24. Zhang X, Li Y, Chen YE, Chen J, Ma PX. Cell-free 3D scaffold with two-stage delivery of miRNA-26a to regenerate critical-sized bone defects. Nat Commun. 2016;7:10376. Jan 14

25. Badieyan ZS, et al. Transcript-activated collagen matrix as sustained mRNA delivery system for bone regeneration. J Control Release. 2016;239:137-48.

26. Zaitseva TS, et al. Aligned nanofibrillar scaffolds for controlled delivery of modified mRNA. Tissue Eng Part A. 2018; https://doi. org/10.1089/ten.TEA.2017.0494.

27. Boczkowski D, Nair SK, Nam JH, Lyerly HK, Gilboa E. Induction of tumor immunity and cytotoxic $\mathrm{T}$ lymphocyte responses using dendritic cells transfected with messenger RNA amplified from tumor cells. Cancer Res. 2000;60:0008-5472.
28. Sunshine J, Green JJ, Mahon KP, Yang F, Eltoukhy AA, Nguyen $\mathrm{DN}$, et al. Small-molecule end-groups of linear polymer determine cell-type gene-delivery efficacy. Adv Mater. 2009;21:4947-51.

29. Marshall AJ, Ratner BD. Quantitative characterization of spheretemplated porous biomaterials. AIChE J. 2005;51:1221-32.

30. Anchordoquy TJ, Carpenter JF, Kroll DJ. Maintenance of transfection rates and physical characterization of lipid/DNA complexes after freeze-drying and rehydration. Arch Biochem Biophys. 1997;348:199-206.

31. Inoh Y, Nagai M, Matsushita K, Nakanishi M, Furuno T. Gene transfection efficiency into dendritic cells is influenced by the size of cationic liposomes/DNA complexes. Eur J Pharm Sci. 2017;102:230-6.

32. Chen R, Ma H, Zhang L, Bryers JD. Precision-porous templated scaffolds of varying pore size drive dendritic cell activation. Biotechnol Bioeng. 2018; 115: 1086-95.

33. Mitchell DA, Nair SK. RNA-transfected dendritic cells in cancer immunotherapy. J Clin Invest. 2000;106:1065-9. Nov

34. Bettinger T, Carlisle RC, Read ML, Ogris M, Seymour LW. Peptide-mediated RNA delivery: a novel approach for enhanced transfection of primary and post-mitotic cells. Nucleic Acids Res. 2001;29:3882-91

35. Rejman J, Tavernier G, Bavarsad N, Demeester J, De Smedt SC. MRNA transfection of cervical carcinoma and mesenchymal stem cells mediated by cationic carriers. J Control Release. 2010;147:385-91.

36. Uchida S, Itaka K, Chen Q, Osada K, Ishii T, Shibata MA, et al. PEGylated polyplex with optimized PEG shielding enhances gene introduction in lungs by minimizing inflammatory responses. Mol Ther. 2012;20:1196-203.

37. Yadava P, Gibbs M, Castro C, Hughes JA. Effect of lyophilization and freeze-thawing on the stability of siRNA-liposome complexes. AAPS PharmSciTech. 2008;9:335-41.

38. Hosseinkhani H, Hosseinkhani M, Gabrielson NP, Pack DW, Khademhosseini A, Kobayashi H. DNA nanoparticles encapsulated in 3D tissue-engineered scaffolds enhance osteogenic differentiation of mesenchymal stem cells. J Biomed Mater Res Part A. $2008 ; 85: 47-60$.

39. Ow-Sullivan MM, Green JJ, Przybycien TM. Development of a novel gene delivery scaffold utilizing colloidal goldpolyethylenimine conjugates for DNA condensation. Gene Ther. 2003;10:1882-90.

40. Raftery RM, Tierney EG, Curtin CM, Cryan SA, O'Brien FJ. Development of a gene-activated scaffold platform for tissue engineering applications using chitosan-pDNA nanoparticles on collagen-based scaffolds. J Control Release. 2015;210:84-94.

41. Capan Y, Woo BH, Gebrekidan S, Ahmed S, DeLuca PP. Preparation and characterization of poly (D,L-lactide-co-glycolide) microspheres for controlled release of poly(L-lysine) complexed plasmid DNA. Pharm Res. 1999;16:509-13.

42. Hosseinkhani H, Azzam T, Kobayashi H, Hiraoka Y, Shimokawa $\mathrm{H}$, Domb AJ, et al. Combination of 3D tissue engineered scaffold and non-viral gene carrier enhance in vitro DNA expression of mesenchymal stem cells. Biomaterials. 2006;27: 4269-78.

43. Pannier AK, Segura T. Surface-and hydrogel-mediated delivery of nucleic acid nanoparticles. Methods Mol Biol. 2013;948: 149-69. 